\title{
A missed syndrome in chronic pelvic pain: Piriformis syndrome
}

\author{
P Y Bahat, ${ }^{1} \mathrm{MD}$; G Turan, ${ }^{2} \mathrm{MD}$; S C Okur, ${ }^{3} \mathrm{MD}$ \\ ${ }^{1}$ Department of Obstetrics and Gynaecology, Kanuni Sultan Süleyman Training and Research Hospital, Istanbul, Turkey \\ ${ }^{2}$ Department of Obstetrics and Gynaecology, Faculty of Medicine, Gazi University, Ankara, Turkey \\ ${ }^{3}$ Physical Medicine and Rehabilitation Unit, Sadi Konuk Training and Research Hospital, Istanbul, Turkey
}

Corresponding author: P Y Bahat (dr_pinaryalcin@hotmail.com)

\begin{abstract}
Piriformis syndrome is a condition that is often overlooked, and that should be considered when assessing chronic pelvic pain. Patients usually describe deeply localised pain in the gluteal region where the piriformis muscle is located. Patients with piriformis syndrome have long-term chronic pelvic pain. The diagnosis and treatment process of such patients is challenging. It can be diagnosed with an accurate examination and anamnesis, and can be treated using various methods.
\end{abstract}

S Afr J Obstet Gynaecol 2019;25(3):72-74. https://doi/org/10.7196/SAJOG.2019.v25i3.1390

Piriformis syndrome is a condition that is often overlooked, and that should be considered when assessing chronic pelvic pain. The purpose of this review is to examine its anatomical and pathophysiological basis, and to review its diagnostic and therapeutic criteria using both a classical approach and a holistic one, including neural therapy.

The syndrome consists of the spread of severe pain from the piriformis muscle to the gluteal region, with or without sciatica symptoms, and the presence of signs and symptoms in this region. The syndrome is often overlooked because its clinical symptoms are similar to other underlying pathologies of lower back pain. Patients usually describe deeply localised pain in the gluteal region, where the piriformis muscle is located. This syndrome is often seen among people aged 40 - 50 years, independent of occupation and activity. It has been reported that the prevalence of piriformis syndrome among patients with chronic low back pain is $5-36 \% \cdot{ }^{[1,2]}$ Piriformis syndrome is six times more common in women than in men. It is thought that the syndrome is caused by biomechanical differences due to a wider angle in women between the quadriceps femoris muscle and the pelvic bone. ${ }^{[2]}$ The piriformis muscle was initially described by the Belgian anatomist Adrian Spigelius in the early 17th century. Calliet, Pace, and Steiner indicated that piriformis muscle injection could be performed. In an article published in 1937, Freiberg ${ }^{[3]}$ reported that sciatic nerve pain could be treated with muscle and fascial surgery, and mentioned the piriformis muscle in the article. The term piriformis syndrome was first used by Robinson ${ }^{[4]}$ in 1947 . The size of the piriformis muscle is variable. It can be small enough that it attaches to the sacrum in only one or two regions, or it may be so large as to spread to the sacroiliac joint (SIJ) capsule, to the anterior surface of the sacrotuberous ligament or even to the bottom of the sacrospinous ligament. The muscle originates at the anterior surface of the sacrum, usually at the levels of vertebrae S2 through S4, at or near the SIJ capsule. On the medial side, it is firmly attached to the protrusions between the first and fourth sacral foramen on the anterior surface of the sacrum. On the lateral side, it is attached to the superior medial surface of the greater trochanter of the femur with a round tendon. It is often combined with the tendons of the obturator internus muscle and the gemelli muscles. It leaves the pelvis through the greater sciatic foramen. The ilium is anterior and superior to the piriformis muscle, the sacrotuberous ligament is posterior, and the sacrospinous ligament is inferior. If the piriformis muscle is larger than normal, this can lead to compression of the blood vessels and nerves of the pelvis. It is innervated with the S1 and S2 sacral nerves and the L5 spinal nerve in general. There are six possible different anatomical relationships between the sciatic nerve and the piriformis muscle where in the: ${ }^{[5-7]}(i)$ sciatic nerve may pass below the piriformis muscle (78 - 98.5\% of the population); (ii) sciatic nerve divisions may pass through and below the piriformis muscle (12-21\%); (iii) sciatic nerve divisions may pass through and above the piriformis muscle; (iv) sciatic nerve divisions may pass above and below the piriformis muscle; $(v)$ sciatic nerve may pass through the piriformis muscle; and ( $v i$ ) sciatic nerve may pass above the piriformis muscle.

The piriformis muscle acts as an external rotator, weak abductor and weak flexor of the hip, providing postural stability during ambulation and standing. In weight-bearing activities, the piriformis muscle restrains vigorous or excessive medial rotation of the thigh. ${ }^{[8-10]}$

\section{Pathophysiology}

Primary causes are associated with anatomical variations (split piriformis muscle, split sciatic nerve and anatomical variations of the sciatic nerve), and these can be seen at a rate of $15 \%$.

Secondary causes (85\%) mostly include macrotraumas and microtraumas to this region and masses, leading to ischaemia. Macrotraumas to this region may cause sciatic nerve compression after soft tissue inflammation and muscle spasm. ${ }^{[11]}$ Conversely, hip pain and sciatica can cause inflammation and spasm of the piriformis muscle. Inflammatory substances secreted from inflamed muscles such as prostaglandin, histamine, bradykinin and serotonin irritate the sciatic nerve and initiate the pain-spasm-pain cycle. Inflammation, spasm or tension in the muscle may cause compression of the sciatic nerve between the piriformis muscle and the pelvis, or between the tendinous region of the piriformis muscle and the bone pelvis. In cases where the piriformis muscle lies anterior to the sciatic nerve, compression occurs between the upper border of the piriformis muscle and the upper border of 
the greater sciatic foramen. In such patients, signs of neurological deficits and positive electrophysiological results can be observed. The sciatic nerve is shortened because scar tissue that develops after laminectomy compresses nerve fibres. Thus, the piriformis muscle becomes prone to repetitive tension and trauma. ${ }^{[12]}$ Microtraumas can develop owing to muscle overuse conditions, such as longdistance walking, running, cycling and rowing. Microtrauma findings can also be seen with repetitive long-standing low pressure (long sitting on hard surfaces, and wallet pressure). ${ }^{[11,12]}$ Weak hip abductors (the gluteus medius) and tight adductor muscles cause the piriformis muscle to shorten and contract strongly. When the external abductor muscles do not function properly, tension would load onto the piriformis muscle. An increase of more than $40 \%$ in the size of the piriformis muscle would lead to compression of the sciatic nerve. A spasm of the piriformis muscle not only compresses the sciatic nerve but also leads to compression of the pudendal nerve. ${ }^{[13]}$ The pudendal nerve controls the bladder and intestinal muscles. Such patients may have tingling and numbness in the groin and buttock areas, and urinary and fecal incontinence. The piriformis muscle may cause gluteal pain spreading to the leg by stimulating the sciatic nerve. If there is involvement of the posterior cutaneous nerve of the thigh, pain may spread from the thigh to the knee. Pain usually increases with longer sitting (driving, cycling), or on standing up from a seated position. Because the piriformis muscle is adjacent to the lateral pelvic wall, pain can occur, with increased bowel movements. Dyspareunia can occur in these patients. There may be a history of limping, scuffing and numbness in the lower part of the leg on the affected side. In the majority of patients, an anterior oblique rotation towards the contralateral side is seen in the sacrum on the side of the affected piriformis muscle (e.g. left oblique rotation and right anterior rotation if there is a problem on the right side). This results in a compensatory rotation towards the affected side in the lower lumbar vertebrae (Fig. 1). TePoorten $^{[14]}$ reports that patients with piriformis syndrome may have a decreased range of motion in the T10 and T11 vertebrae, tissue structure changes and a decreased range of motion in the T3 and T4 vertebrae, pain and decreased range of motion at the $\mathrm{C} 2$ level on the contralateral side, and lesions in the atlanto-occipital transition on the ipsilateral side.

Three factors must be considered with regard to pain caused by piriformis syndrome: (i) referred pain due to trigger point development in the piriformis muscle; (ii) sciatica due to irritation of the piriformis muscle; and (iii) SIJ dysfunction. ${ }^{[10]}$

As is known, spasm, trigger point and tension problems in muscle

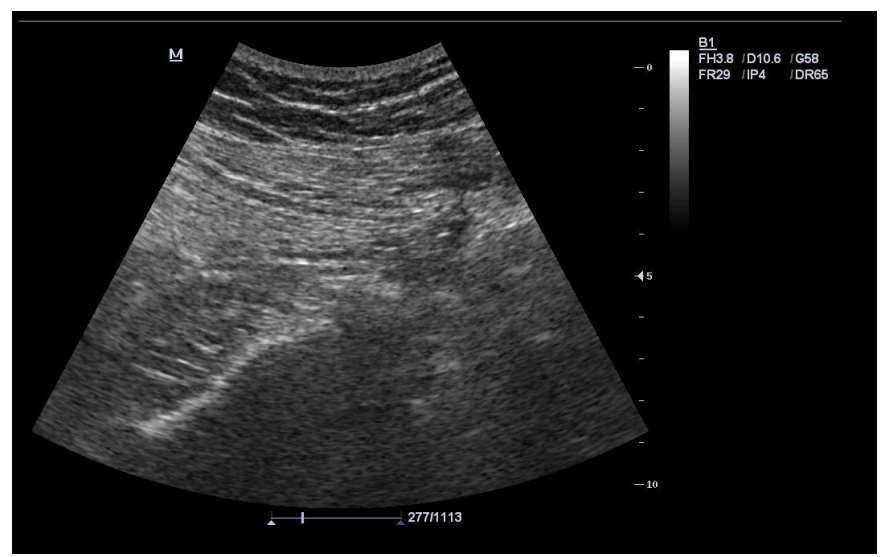

Fig. 1 Appearance of priformis muscle injection under ultrasound. occur as a result of latent acidosis and lymphatic dysfunction due to impaired perfusion and unremoved degradation products. In the case of continuation of latent acidosis caused by degradation products resulting from inflammation, this increases the body burden and prepares the ground for vegetative burden and the deterioration of the systemic structure. ${ }^{[14]}$

\section{Symptoms}

Pain can occur when standing, sitting or lying down in the same position for 15 - 20 minutes. Pain spreading from the sacrum into the gluteal region and generally the knee can occur. It can mimic radicular pain owing to irritation of the sciatic nerve. Pain increases with movement, and decreases with rest. When standing up from a seated position or when squatting, pain increases. It does not completely disappear with position change. Pain may occur in the contralateral SIJ. Walking difficulty (antalgic gait, drop foot) can occur, as well as foot numbness and weakness in the lower extremity on the affected side, headache and neck pain, pain in the abdominal, pelvic and inguinal regions. Dyspareunia may occur in women. Pain increases with bowel movements.

\section{Examination findings}

The following may be found:

- tenderness with palpation in the SIJ, greater sciatic foramen and piriformis muscle

- tenderness over the piriformis muscle

- palpable mass at the anatomical location of the piriformis muscle

- reduction of pain on applying traction to the problematic leg

- asymmetric weakness in the problematic leg

- Pace test (FAIR (flexion, adduction, internal rotation)) positive

- Lasegue test positive

- Freiberg test positive

- Beatty test positive

- internal rotation of the lower extremity on the affected side restricted

- short leg on the affected side

- gluteal atrophy (in chronic cases)

- compensatory lumbar rotation with permanent sacral rotation towards the contralateral side.

The causes of lower back pain and sciatica should be considered in the differential diagnosis. Patients with piriformis syndrome who do not usually have neurological deficits are assessed as herniated nucleus pulposus, and thus misdiagnosed. Other conditions that cause spinal stenosis, facet syndrome, SIJ dysfunction, trochanteric bursitis, myofascial pain, pelvic tumour, endometriosis and sciatic nerve irritation should be considered.

\section{Treatment}

Non-invasive methods are preferred in the treatment of piriformis syndrome. Paracetamol and nonsteroidal anti-inflammatory drugs are recommended for pain control. Particular exercises should be added to the treatment as an absolute requirement. Piriformis stretching, sacroiliac mobilisation hamstring stretching and pelvic floor muscle exercises should be included in treatment programmes. The entire protocol should be organised as 10 repetitions, twice a day. Electrotherapy, transcutaneous electrical nerve stimulation and low-level laser treatments are physical therapy modalities that can be applied to increase blood flow. 


\section{Manual therapy techniques}

Manipulation and mobilisation of the SIJ on the affected side, lumbar manipulation, piriformis stretching exercises and quadratus lumborum stretching and relaxing exercises are effective treatment modalities that can be applied for aetiological causes. Vaginal and rectal manual examination for the pelvic floor and manipulation techniques applied to trigger points detected in the levator ani muscle also give important information on the assessment and treatment of the pelvic floor and related structures. ${ }^{[15,16]}$

\section{Piriformis muscle injection}

In cases such as trigger point and piriformis spasm that are not resolved by systemic drug use, piriformis muscle injection is frequently used, both to clarify diagnosis and to provide treatment.

\section{Ultrasonographic technique}

The patient is placed in the prone position. A convex transducer (2 - $5 \mathrm{MHz}$ ) is placed transversally on the $\mathrm{S} 1$ spinous process. The transducer is moved slightly laterally and caudally. The spina iliaca posterior superior (SIPS) and gluteus maximus are visualised. Then, the transducer is moved further laterally and caudally to visualise the piriformis muscle on the iliac bone. Isolated movement of the piriformis muscle is observed with passive rotation of the hip. The sciatic nerve is visualised in the mediocaudal area of the piriformis muscle. Piriformis muscle injection is performed with a 22 -gauge needle using the in-plane technique. Medications such as $0.5 \%$ lidocaine, triamcinolone acetonide and botulinum toxin A can be used for injection. If needed, SIJ injection can also be included in the treatment. ${ }^{[17,18]}$

\section{Blind technique}

The patient is placed in the prone position. The distance between the greater trochanter and the SIPS is divided into two equal parts. The precise trigger point is fixed between two fingers, and the injection is performed. To avoid contact with the sciatic nerve in this region, the needle should be inserted laterally into the piriformis muscle (the greater trochanter and SIPS is divided into three equal parts, and the injection is performed medially from the lateral third). During advancement of the needle, the person injecting should remain in communication with the patient and should question whether there is a feeling of electric shock or numbness. It is expected to reach the muscle at a depth of $\sim 2.5-5.0 \mathrm{~cm}$. Sciatic nerve penetration is the most feared complication in this injection. The procedure should therefore be performed by an experienced physician or someone experienced in the technique. ${ }^{[19]}$

In a cadaver study conducted to determine the exact placement of the sciatic nerve in this region, it was found that the mean (standard deviation; range) distance between the lower edge of the SIJ and the sciatic nerve was $2.9(0.6 ; 1.8-3.7) \mathrm{cm}$ laterally and $0.7(0.7 ; 0-2.5) \mathrm{cm}$ caudally. The width of the sciatic nerve was measured as $1.5(0.3 ; 1-3.5) \mathrm{cm}$.

In operations performed under fluoroscopy, the needle is vertically inserted from the midpoint of the distance between the great trochanter and the SIPS. The spread of contrast medium within the muscle is observed, and then local anesthetic injection is performed. ${ }^{[20]}$ Rarely, surgical release of the piriformis muscle in difficult cases is among the treatment options. ${ }^{[21]}$

\section{Neural therapy approach for piriformis syndrome}

Neural therapy is a complementary medicine method in which injection techniques are used. It is often used in musculoskeletal problems. Lidocaine and procaine are commonly used medications. Segment therapy begins with subcutaneous injection to the T9 S4 segments in piriformis syndrome. Scar tissues in the segments are also irrigated by the injection. Trigger point injection to the piriformis muscle and SIJ injection are included in the treatment.

Head's zones, Fankhauser ganglion or prostate injection (to reach the anterior fibres of the sacral plexus and to regulate the urogenital organs within the segment), sacral canal injection, opening of the lymphatic drainage of the lower extremity and sympathetic ganglion blockade at the L2 level on the affected side are included in the treatment. ${ }^{[22,23]}$

\section{Acknowledgements. None.}

Author contributions. PYN and GT contributed substantially to the conception and design of the study. PYN and GT contributed to the acquisition of data. SC contributed to the analysis and interpretation. PYC drafted the article. PYC and SC provided critical revision of the article. PYC provided final approval of the version to publish.

Funding. None.

Conflicts of interest. None.

1. Foster MR. Piriformis syndrome. Orthopedics 2002;25(8):821-825. https://doi.org/10.3928/0147 7447-20020801-12

2. Pace JB, Nagle D. Piriformis syndrome. West J Med 1976;124:435-439.

3. Freiburg AH. Sciatic pain and its relief by operations on muscle and fascia. Arch Surg 1937;34(2):337-350. https://doi.org/10.1001/archsurg.1937.01190080138007

4. Robinson DR. Pyriformis syndrome in relation to sciatic pain. Am J Surg 1947;73(3):355-358.

5. Parziale JR, Hudgins TH, Fishman LM. The piriformis syndrome. Am J Orthop 1996;25:819-813.

6. Beason LE, Anson BJ. The relation of the sciatic nerve and its subdivisions to the piriformis muscle. Anat Record 1937;70:1-5. https://doi.org/10.1002/ar.1090700102

7. Pecina M. Contribution to the etiological explanation of the piriformis syndrome. Acta Anat 1979;105(2):181-187. https://doi.org/10.1159/000145121

8. Papadopoulos EC, Khan SN. Piriformis syndrome and low back pain: A new classification and review of the literature. Orthop Clin North Am 2004;35(1):65-71. https://doi.org/10.1016/s00305898(03)00105-6

9. Williams PL, Warwick R. Gray's Anatomy. 36th ed. Philadelphia: WB Saunders Co., 1980.

10. Raj PP. Radiographic Imaging for Regional Anesthesia and Pain Management. 1st ed. Philadelphia: Churchill Livingstone, 2003.

11. Boyajian-O'Neil LA, McClain RL, Coleman MK, Thomas PP. Diagnosis and management of piriformis syndrome: An osteopathic approach. JAOA 2008;108:657664. https://doi.org/10.7556/

12. Bezon HT, Katz JA. Piriformis syndrome. Anesthesiology 2003;98(6):1442-1448. https://doi. org/10.1097/00000542-200306000-00022

13. Retzlaff E, Berry AH, Haight AS, et al. The piriformis muscle syndrome. JAOA 1974;73(10):799-807.

14. TePoorten BA. The piriformis muscle. JAOA 1969:69:150-160.

15. Rashbaum RF, Ohnmeiss DD, Lindley EM, Kitchel SH, Patel VV. Sacroiliac joint pain and its treatment. Clin Spine Surg 2016;29(2):42-48. https://doi.org/10.1097/bsd.0000000000000359

16. Jonely H, Brismée JM, Desai MJ, Reoli R. Chronic sacroiliac joint and pelvic girdle dysfunction in a 35 -year-old nulliparous woman successfully managed with multimodal and multidisciplinary approach. J Man Manip Ther 2015;23(1):20-26. https://doi.org/10.1179/2042618614Y.0000000086

17. Bevilacqua Alén E, Diz Villar A, Curt Nuño F, et al. Ultrasound-guided piriformis muscle injection. A new approach. Rev Esp Anestesiol Reanim 2016;63(10):594-598. https://doi.org/10.1016/j. redar.2016.02.008

18. Santamato A, Micello MF, Valeno G, et al. Ultrasound-guided injection of botulinum toxin type a for piriformis muscle syndrome: A case report and review of the literature. Toxins 2015;7(8):3045-3056 piriformis muscle syndrome: A case report
https://doi.org/ $10.3390 \% 2$ Ftoxins 7083045

19. Chang A, Dulebohn SC. Piriformis Injection. Treasure Island (FL): StatPearls Publishing, 2020.

20. Kirschner JS, Foye PM, Cole JL. Piriformis syndrome, diagnosis and treatment. Muscle Nerve 2009;40(1):10-18. https://doi.org/10.1002/mus.21318

21. H. Nazlıkul. Nöralterapi. Istanbul: Nobel Tip Kitabevi, 2010.

22. Anderson RU. Traditional therapy for chronic pelvic pain does not work: What do we do now? Nature Clin Pract Urology 2006;3:145-56. https://doi.org/10.1038/ncpuro0438

23. Anderson RU, Sawyer T, Wise D, Morey A, Nathanson BH. Painful myofascial trigger points and pain sites in men with chronic prostatitis/chronic pelvic pain syndrome. J Urol 2009;182(6):2753-2758. https://doi.org/10.1016/j.juro.2009.08.033

Accepted 16 February 2020. 